TITLE:

\title{
Portfolio Allocation Problems between Risky and Ambiguous Assets
}

$\operatorname{AUTHOR}(S)$ :

Asano, Takao; Osaki, Yusuke

\section{CITATION:}

Asano, Takao ...[et al]. Portfolio Allocation Problems between Risky and Ambiguous Assets. KIER Discussion Paper 2017, 975: 1-25

ISSUE DATE:

2017-08-28

URL:

http://hdl.handle.net/2433/226996

RIGHT: 


\title{
KIER DISCUSSION PAPER SERIES
}

\author{
KYOTO INSTITUTE \\ OF \\ ECONOMIC RESEARCH
}

\author{
Discussion Paper No.975 \\ "Portfolio Allocation Problems between Risky \\ and Ambiguous Assets"
}

Takao Asano Yusuke Osaki

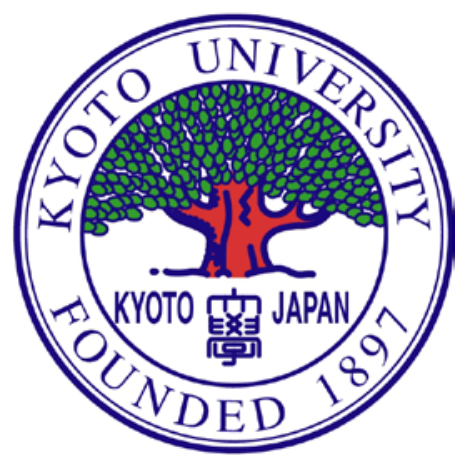

KYOTO UNIVERSITY

KYOTO, JAPAN 


\title{
Portfolio Allocation Problems between Risky and Ambiguous Assets*
}

\author{
Takao Asano ${ }^{\dagger}$ Yusuke Osaki ${ }^{\ddagger}$ \\ This Version: August 28, 2017
}

\begin{abstract}
This paper considers a portfolio allocation problem between a risky asset and an ambiguous asset, and investigates how the existence of ambiguity influences the optimal proportion invested in the two assets. By introducing the notion of ambiguity, we derive several sufficient conditions under which an investor decreases the optimal proportion invested in the ambiguous asset. Furthermore, as an application, we consider an international diversification problem, and show that the home bias puzzle is partially resolved.
\end{abstract}

JEL Classification Numbers: G11; D81

Key Words: Home Bias Puzzle, Portfolio Allocation Problem, Smooth Ambiguity Model

*We are grateful to Masamitsu Ohnishi and participants at the 2017 Annual Meeting of the Nippon Finance Association. Needless to say, we are responsible for any remaining errors. This research is financially supported by the JSPS KAKENHI Grant Numbers 26380240, 26380411 and 26705004, and the Joint Research Program of KIER.

${ }^{\dagger}$ Faculty of Economics, Okayama University, 3-1-1, Tsushimanaka, Kita-ku, Okayama 700-8530, Japan. e-mail: asano@e.okayama-u.ac.jp

${ }^{\ddagger}$ Faculty of Economics, Osaka Sangyo University, 3-1-1, Nakagaito, Daito, Osaka 5748530, Japan. e-mail: osaki@eco.osaka-sandai.ac.jp 


\section{Introduction}

In the real world, it is difficult to precisely predict what will happen in the future. In particular, in financial markets, it is difficult for investors to accurately foresee returns on assets. Therefore, it is worth investigating how investors diversify their wealths across different assets under uncertainty. The notion of uncertainty $^{1}$ has been investigated in the literature since Keynes (1921) and Knight (1921). While risk is a situation in which the beliefs of a decision maker (DM) are captured by a unique probability measure, ambiguity is a situation in which a DM's beliefs are not pinned down by a unique probability measure because of a lack of information. In previous research, portfolio selection problems have been analyzed within the frameworks of expected utility theory and non-expected utility theories. While investors are supposed to allocate their wealths to a safe asset $^{2}$ and a risky asset under expected utility theory, ${ }^{3}$ they are assumed to allocate their wealths to a safe asset and an ambiguous asset $^{4}$ under non-expected utility theories. ${ }^{5}$ Although these analyses clarify the effects of risk or ambiguity on portfolio selection problems, in financial markets, it is appropriate to analyze situations in which investors consider a risky asset and an ambiguous asset simultaneously. Therefore, by incorporating the notions of both risk and ambiguity into portfolio selection problems as well as introducing some notions of stochastic dominance to capture shifts in returns on assets, ${ }^{6}$ we investigate how the existence of ambiguity affects optimal portfolio allocation problems.

As explained above, the notion of ambiguity is suitable for capturing situa-

\footnotetext{
${ }^{1}$ Following Strazalecki (2013), we term uncertainty as an umbrella term for both risk and ambiguity.

${ }^{2}$ Throughout this paper, to avoid confusion, we say that an asset whose return is known with certainty is safe, rather than riskless or risk-free.

${ }^{3}$ For example, see Hadar and Seo $(1988,1990)$ as mentioned in Introduction and subsection 4.1 .

${ }^{4}$ Throughout this paper, we say that an asset whose return is captured by a unique probability measure is risky and an asset whose return is not captured by a unique probability measure is ambiguous.

${ }^{5}$ See Gollier (2011).

${ }^{6}$ For a survey of stochastic dominance, see Levy (1992).
} 
tions in which investors possess different information about returns on assets. This enables us to analyze a portfolio allocation problem between a risky asset and an ambiguous asset. For example, let us consider an investor who plans to purchase equities in her local and foreign markets. It is natural to assume that she confronts more difficulty predicting returns on foreign equities than on local equities because of the difference in information. Moreover, a portfolio consisting of risky and ambiguous assets is more general than a portfolio consisting of safe and risky assets or safe and ambiguous assets. In this situation, returns on foreign equities are more uncertain for the investor than those on local equities, which is captured appropriately by risk and ambiguity. As another example, suppose that a firm determines the budgets for existing and new businesses. It is appropriate to assume that the profit from the former is more predictable than that from the latter. Therefore, we consider the profit from existing businesses to be captured by risk and the profit from new ones to be captured by ambiguity.

Ellsberg (1961) shows experimentally that DMs typically dislike situations where they cannot assign a unique probability measure. This behavior, which is called ambiguity aversion, cannot be explained in the framework of expected utility theory. To overcome the shortcomings of expected utility theory pointed out by Ellsberg (1961), many preference representations, which is called as ambiguity models, have been proposed. For example, Gilboa and Schmeidler (1989) propose max-min expected utility theory (MEU), and Schmeidler (1989) proposes Choquet expected utility theory (CEU). In this paper, we adopt the smooth ambiguity model by Klibanoff et al. (2005) as our ambiguity model. This is because the smooth ambiguity model can differentiate the DMs' attitude towards ambiguity from their perception of ambiguity, which implies that the smooth ambiguity model is more general than MEU and CEU. Furthermore, because the smooth ambiguity model has a "double" expected utility form, it is more tractable than most of ambiguity models.

Several studies in the literature on portfolio selection problems are worth mentioning. Hadar and Seo $(1988,1990)$ derive conditions on utility functions when returns on assets are shifted by first-order stochastic dominance (FSD). Conditions on utility functions can be removed by concepts stronger 
than FSD, such as monotone likelihood ratio dominance (MLRD) by Landsber and Meilijson (1990) and reversed hazard ratio dominance (RHRD) by Kijima and Ohnishi (1996). ${ }^{7}$ Kijima and Ohnshi (1996) provide a systematic method for stochastic dominance that is useful for portfolio allocation problems. While these papers analyze portfolio selection problems within the framework of expected utility theory, Gollier (2011), Osaki and Schlesinger (2014), and this paper investigate portfolio selection problems within the framework of the smooth ambiguity model. Gollier (2011) introduces ambiguity into returns on an asset and derives sufficient conditions under which any increase in ambiguity aversion decreases the purchase of the ambiguous asset. While Gollier (2011) analyzes portfolios consisting of one safe asset and one ambiguous asset, we analyze portfolios consisting of one risky asset and one ambiguous asset. As mentioned in the above example, in financial markets, it is appropriate to analyze portfolios consisting of one risky asset and one ambiguous asset. Osaki and Schlesinger (2014) do not introduce ambiguity into returns on an asset, but investigate background uncertainty, which cannot be controlled or traded. As in Osaki and Schlesinger (2014), we consider situations with different levels of uncertainty. However, we introduce ambiguity into our model as tradable uncertainty, while in Osaki and Schlesinger (2014) ambiguity is non-tradable.

The organization of this paper is as follows. Section 2 formulates a portfolio allocation problem between a risky asset and an ambiguous asset. Section 3 states the main result of this paper and discusses an implication for the home bias puzzle. Section 4 provides further results applying the main theorem of this paper. Section 5 concludes this paper. Some of the mathematical definitions and proofs are relegated to the Appendix.

\footnotetext{
${ }^{7}$ Reversed hazard ratio dominance is also called as monotone probability ratio dominance. See Eeckhoudt and Gollier (1995).
} 


\section{Portfolio Allocation Problem with Two Un- certain Assets}

In this section, we present a portfolio allocation problem based on the smooth ambiguity model by Klibanoff et al. (2005) in which an investor is faced with both risk and ambiguity.

To simultaneously analyze the effects of risk and ambiguity on portfolio choices, we consider an investor who allocates her wealth $w$ between two uncertain assets, a risky asset and an ambiguous asset. ${ }^{8}$ The return on the risky asset is denoted by the random variable $\tilde{x}$ whose probability distribution function $F$ is defined over the bounded support $[a, b]$ with $a<0<b$. The ambiguity of the return is represented by the second-order probability approach based on Segal (1987) and Klibanoff et al. (2005). There are $n$ possibilities of the return on the ambiguous asset which are indexed by $\theta \in \Theta=\{1, \ldots, n\}$. The possible returns on the ambiguous asset are denoted by $\tilde{y}_{\theta}, \theta=1, \ldots, n$. The probability distribution function of $\tilde{y}_{\theta}$ is denoted by $G_{\theta}$ and is defined over the bounded support $[a, b]$. For simplicity, all possible probability distribution functions are assumed to be defined over the same support $[a, b]$. The investor attaches the second-order probability $\left\{q_{1}, \ldots, q_{n}\right\}$ to the index set $\Theta$. The return on the risky asset $\tilde{x}$ and the possible returns on the ambiguous asset $\tilde{y}_{\theta}$ are assumed to be independent. The investor's preferences are assumed to be represented by the smooth ambiguity model by Klibanoff et al. (2005).

The investor chooses her portfolio allocation $(w-k, k)$ to maximize the welfare from the terminal wealth. Here, $w-k$ is the amount invested in the risky asset and $k$ is the amount invested in the ambiguous asset. Her objective is to maximize the following:

$$
V(k)=\sum_{\theta=1}^{n} q_{\theta} \phi\left(E\left[u\left((w-k) \tilde{x}+k \tilde{y}_{\theta}\right)\right]\right) .
$$

We assume that $u$ is strictly increasing and strictly concave, that is, $u^{\prime}>0$ and $u^{\prime \prime}<0$, and $\phi$ is strictly increasing and concave, that is, $\phi^{\prime}>0$ and

\footnotetext{
${ }^{8}$ In the literature, for example, Gollier (2011) and Huang and Tzeng (2017) consider one safe asset and one ambiguous asset.
} 
$\phi^{\prime \prime} \leq 0$. The attitude towards ambiguity is captured by the curvature of $\phi$. The concavity captures an investor's ambiguity aversion in the sense that she dislikes any mean-preserving spread of the expected utility of $u$. The linearity of $\phi$ captures her ambiguity neutrality in the sense that ambiguity degenerates to the single return on the asset, $\tilde{y}_{O} \stackrel{\mathrm{d}}{=} \sum_{\theta=1}^{n} q_{\theta} \tilde{y}_{\theta}$, where $\stackrel{\mathrm{d}}{=}$ indicates equality in distribution. The linearity of $\phi$ plays the benchmark role for the following analyses.

The optimal portfolio allocation $k^{*}$ is the solution of the following firstorder condition (FOC):

$V^{\prime}\left(k^{*}\right)=\sum_{\theta=1}^{n} q_{\theta} \phi^{\prime}\left(E\left[u\left(\left(w-k^{*}\right) \tilde{x}+k^{*} \tilde{y}_{\theta}\right)\right]\right) E\left[\left(\tilde{y}_{\theta}-\tilde{x}\right) u^{\prime}\left(\left(w-k^{*}\right) \tilde{x}+k^{*} \tilde{y}_{\theta}\right)\right]=0$.

The second-order condition is satisfied by the concavities of $u$ and $\phi$. We suppose that $V^{\prime}(0)>0$ and $V^{\prime}(w)<0$, that is, the investor allocates a positive amount of her wealth to each asset.

We define $k^{\theta}$ as follows:

$$
\left.k^{\theta}=\operatorname{argmax}_{k} E\left[u\left((w-k) \tilde{x}+k \tilde{y}_{\theta}\right)\right]\right), \theta=1, \ldots, n,
$$

where $k^{\theta}$ denotes the ex-post optimal portfolio allocation given $\theta$. When we set $\tilde{y}_{\theta} \stackrel{\mathrm{d}}{=} \tilde{y}_{O}$, the optimal portfolio allocation is denoted by $k^{O}$. This value $k^{O}$ corresponds to the optimal portfolio allocation for an ambiguity neutral investor and is equal to the optimal portfolio allocation for an expected utility maximizer. In other words, ambiguity disappears in this case and the investor faces two risky assets. In the following sections, we examine the effects of ambiguity on the optimal portfolio allocations by comparing $k^{*}$ with $k^{O}$.

\section{Effects of Ambiguity on Portfolio Alloca- tions}

In this section, we provide the main result of this paper and an informal proof. A formal proof is relegated to Appendix B. We then analyze the home bias puzzle as an implication of the result. 


\subsection{The Main Result}

We introduce some definitions and notation before stating the main result. To obtain a clear result, we consider the situation in which the possible returns on the ambiguous asset are ranked by FSD. Let $\tilde{y}_{i}$ and $\tilde{y}_{j}$ be random variables for $i, j \in \Theta=\{1, \ldots, n\}$. We say that $\tilde{y}_{j}$ is greater than $\tilde{y}_{i}$ in the sense of FSD, denoted by $\tilde{y}_{i} \lesssim_{\mathrm{FSD}} \tilde{y}_{j}$, if $G_{i}(y) \geq G_{j}(y)$ holds for any $y \in[a, b]$ and every $i, j \in \Theta$ with $i<j$, where $G_{\theta}$ denotes the probability distribution function of $\tilde{y}_{\theta}$ for $\theta \in \Theta$. The Arrow-Pratt measure of relative risk aversion is defined by $R(z)=-z u^{\prime \prime}(z) / u^{\prime}(z) .{ }^{9}$ This terminology is used within the framework of expected utility theory. We also use it in the smooth ambiguity model. The following result shows that under some conditions, the existence of ambiguity decreases the optimal amount of investment compared with the case in which ambiguity does not exist.

Theorem 1. The existence of ambiguity aversion decreases the optimal portfolio allocation, $k^{*} \leq k^{O}$, if the possible returns on an ambiguous asset $\left\{\tilde{y}_{1}, \ldots, \tilde{y}_{n}\right\}$ are ranked by FSD and $R(z) \leq 1$.

Proof. The proof is relegated to Appendix B.

For comparison, consider the situation in which there are two risky assets whose returns are denoted by $\tilde{x}$ and $\tilde{y}$. In this situation, the investor should allocate $w-k^{O}$ to the risky asset $\tilde{x}$ and $k^{O}$ to the risky asset $\tilde{y}$. For the purpose of introducing ambiguity, consider the situation in which $\tilde{x}$ denote the return on the risky asset and $\tilde{y}$ denotes the return on the ambiguous asset. Therefore, the possible returns on the ambiguous asset are denoted by $\left\{\tilde{y}_{1}, \ldots, \tilde{y}_{n}\right\}$. In this situation, the investor should allocate $w-k^{*}$ to the risky asset and $k^{*}$ to the ambiguous asset. That is, the investor increases the portfolio allocation to the risky asset, or equivalently, decreases the allocation to the ambiguous asset, compared with the case in which both assets are risky. This is because an investor with insufficient information about the return on the ambiguous asset is likely to avoid to invest in the ambiguous asset.

\footnotetext{
${ }^{9}$ See Arrow (1965) and Pratt (1964).
} 
To gain intuitive understanding, we consider the following example in which there are only two indices $\Theta=\{1,2\}$. The FOC can be written by using the Radon-Nikodym derivatives $\left\{\hat{q}_{1}, \hat{q}_{2}\right\}$ :

$$
\begin{aligned}
V^{\prime}\left(k^{*}\right) & =\hat{q}_{1} E\left[\left(\tilde{y}_{1}-\tilde{x}\right) u^{\prime}\left(\left(w-k^{*}\right) \tilde{x}+k^{*} \tilde{y}_{1}\right)\right] \\
& +\hat{q}_{2} E\left[\left(\tilde{y}_{2}-\tilde{x}\right) u^{\prime}\left(\left(w-k^{*}\right) \tilde{x}+k^{*} \tilde{y}_{2}\right)\right]=0,
\end{aligned}
$$

where

$$
\hat{q}_{\theta}=\frac{q_{\theta} \phi^{\prime}\left(E\left[u\left(\left(w-k^{*}\right) \tilde{x}+k^{*} \tilde{y}_{\theta}\right)\right]\right)}{q_{1} \phi^{\prime}\left(E\left[u\left(\left(w-k^{*}\right) \tilde{x}+k^{*} \tilde{y}_{1}\right)\right]\right)+q_{2} \phi^{\prime}\left(E\left[u\left(\left(w-k^{*}\right) \tilde{x}+k^{*} \tilde{y}_{2}\right)\right]\right)}, \theta=1,2 .
$$

Without loss of generality, we assume that $E\left[u\left(\left(w-k^{*}\right) \tilde{x}+k^{*} \tilde{y}_{1}\right)\right] \leq E[u((w-$ $\left.\left.\left.k^{*}\right) \tilde{x}+k^{*} \tilde{y}_{2}\right)\right]$. We can then show that ambiguity averse investors put more weight on a lower expected utility than on a higher expected utility because $\phi^{\prime}$ is decreasing. That is,

$$
\begin{aligned}
& E\left[u\left(\left(w-k^{*}\right) \tilde{x}+k^{*} \tilde{y}_{1}\right)\right] \leq E\left[u\left(\left(w-k^{*}\right) \tilde{x}+k^{*} \tilde{y}_{2}\right)\right] \\
&\left.\Leftrightarrow \quad \hat{q}_{1} \geq q_{1} \text { (equivalently, } \hat{q}_{2} \leq q_{2} \text { because } \hat{q}_{1}=1-\hat{q}_{2} \text { and } q_{1}=1-q_{2}\right) .(3)
\end{aligned}
$$

Note that $\hat{q}_{\theta}=q_{\theta}$ if $\phi$ is linear. In this case, $\hat{q}_{\theta}$ is not distorted, which implies that this kind of investors does not take into accout the existence of ambiguity. Thus, (2) can be written as

$$
\begin{aligned}
V^{\prime}\left(k^{O}\right) & =q_{1} E\left[\left(\tilde{y}_{1}-\tilde{x}\right) u^{\prime}\left(\left(w-k^{O}\right) \tilde{x}+k^{O} \tilde{y}_{1}\right)\right] \\
& +q_{2} E\left[\left(\tilde{y}_{2}-\tilde{x}\right) u^{\prime}\left(\left(w-k^{O}\right) \tilde{x}+k^{O} \tilde{y}_{2}\right)\right] \\
& =E\left[\left(\tilde{y}_{O}-\tilde{x}\right) u^{\prime}\left(\left(w-k^{O}\right) \tilde{x}+k^{O} \tilde{y}_{O}\right)\right]=0,
\end{aligned}
$$

when $\phi$ is linear. Recall that the linearity of $\phi$ corresponds to both assets being risky. It follows from (4) that the sign of $E\left[\left(\tilde{y}_{\theta}-\tilde{x}\right) u^{\prime}\left(\left(w-k^{O}\right) \tilde{x}+k^{O} \tilde{y}_{\theta}\right)\right]$ is different for $\theta=1$ and $\theta=2$ unless $E\left[\left(\tilde{y}_{\theta}-\tilde{x}\right) u^{\prime}\left(\left(w-k^{O}\right) \tilde{x}+k^{O} \tilde{y}_{\theta}\right)\right]=0$. That is, either

$$
E\left[\left(\tilde{y}_{1}-\tilde{x}\right) u^{\prime}\left(\left(w-k^{O}\right) \tilde{x}+k^{O} \tilde{y}_{1}\right)\right] \leq 0 \leq E\left[\left(\tilde{y}_{2}-\tilde{x}\right) u^{\prime}\left(\left(w-k^{O}\right) \tilde{x}+k^{O} \tilde{y}_{2}\right)\right]
$$

or

$$
E\left[\left(\tilde{y}_{1}-\tilde{x}\right) u^{\prime}\left(\left(w-k^{O}\right) \tilde{x}+k^{O} \tilde{y}_{1}\right)\right] \geq 0 \geq E\left[\left(\tilde{y}_{2}-\tilde{x}\right) u^{\prime}\left(\left(w-k^{O}\right) \tilde{x}+k^{O} \tilde{y}_{2}\right)\right] .
$$


For the former case, it follows by combining (3) and (5) that

$$
\begin{aligned}
0 & =V^{\prime}\left(k^{O}\right) \\
& =q_{1} E\left[\left(\tilde{y}_{1}-\tilde{x}\right) u^{\prime}\left(\left(w-k^{O}\right) \tilde{x}+k^{O} \tilde{y}_{1}\right)\right]+q_{2} E\left[\left(\tilde{y}_{2}-\tilde{x}\right) u^{\prime}\left(\left(w-k^{O}\right) \tilde{x}+k^{O} \tilde{y}_{2}\right)\right] \\
& \geq \hat{q_{1}} E\left[\left(\tilde{y}_{1}-\tilde{x}\right) u^{\prime}\left(\left(w-k^{O}\right) \tilde{x}+k^{O} \tilde{y}_{1}\right)\right]+\hat{q_{2}} E\left[\left(\tilde{y}_{2}-\tilde{x}\right) u^{\prime}\left(\left(w-k^{O}\right) \tilde{x}+k^{O} \tilde{y}_{2}\right)\right] \\
& =V^{\prime}\left(k^{*}\right) \\
& \Leftrightarrow k^{*} \leq k^{O} .
\end{aligned}
$$

This is an intuitive case in which the existence of ambiguity decreases the amount invested in the ambiguous asset. This result follows from (5), which states that investors increase their portfolio allocations for higher expected utility. To explain this investment behavior, it seems natural to suppose that (5) holds. However, as shown by, for example, Hadar and Seo (1990), it is well known that such a condition does not necessarily hold. Therefore, additional conditions are required to show that the existence of ambiguity decreases the optimal portfolio allocation. Theorem 1 provides one such sufficient condition. In the following section, we present other sufficient conditions.

For the latter case, it follows by combining (3) and (6) that

$$
\begin{aligned}
0 & =V^{\prime}\left(k^{O}\right) \\
& =q_{1} E\left[\left(\tilde{y}_{1}-\tilde{x}\right) u^{\prime}\left(\left(w-k^{O}\right) \tilde{x}+k^{O} \tilde{y}_{1}\right)\right]+q_{2} E\left[\left(\tilde{y}_{2}-\tilde{x}\right) u^{\prime}\left(\left(w-k^{O}\right) \tilde{x}+k^{O} \tilde{y}_{2}\right)\right] \\
& \leq \hat{q_{1}} E\left[\left(\tilde{y}_{1}-\tilde{x}\right) u^{\prime}\left(\left(w-k^{O}\right) \tilde{x}+k^{O} \tilde{y}_{1}\right)\right]+\hat{q_{2}} E\left[\left(\tilde{y}_{2}-\tilde{x}\right) u^{\prime}\left(\left(w-k^{O}\right) \tilde{x}+k^{O} \tilde{y}_{2}\right)\right] \\
& =V^{\prime}\left(k^{*}\right) \\
& \Leftrightarrow k^{*} \geq k^{O} .
\end{aligned}
$$

This is a counterintuitive case in which the existence of ambiguity increases the amount invested in the ambiguous asset.

As a final remark, we discuss the assumption of the independence. Kijima and Ohnishi (1996) show that Inequalities (5) hold for FSD even though the return on the risky asset $\tilde{x}$ and each possible return on the ambiguous asset $\tilde{y}_{\theta}$ are dependent, not independent. However, the convolution property cannot be guaranteed to hold. ${ }^{10}$ If this property does not hold, Inequality (3) may

\footnotetext{
${ }^{10}$ For the definition of the convolution property, see Appendix A.
} 
be reversed, that is, it is possible that $\hat{q}_{1}<q_{1}$. In this case, we obtain the counterintuitive result that states that the existence of ambiguity increases the amount invested in the ambiguous asset. On the other hand, if the convolusion property holds, we obtain Theorem 1 in the case of the dependence.

\subsection{The Home Bias Puzzle}

In this subsection, we apply Theorem 1 to an international diversification problem, which provides a solution to the home bias puzzle from the viewpoint of ambiguity.

French and Poterba (1991) observe the tendency for investors to hold more equities in their home country than in their foreign countries, which is contrary to theoretical results obtained from macroeconomic models. This is called the home bias puzzle. This puzzle cannot be explained by standard macroeconomic models within the framework of expected utility theory. ${ }^{11}$ It is natural to assume that investors have more information about assets in their home country than those in foreign countries. Therefore, ambiguity may play an important role in explaining the home bias puzzle. ${ }^{12}$ We investigate how the difference between individual investors with insufficient information and institutional investors with much information explains the home bias puzzle.

Let us consider an ambiguity averse individual investor who allocates her wealth $w$ between a domestic asset and a foreign asset. The investor possesses enough information to quantify the return on the domestic asset using a single probability distribution, but she does not have enough information to quantify the return on the foreign asset similarly. In this situation, the domestic asset is risky and the foreign asset is ambiguous. This setting is the same as in the previous section. The return on the domestic asset is denoted by $\tilde{x}$, and the return on the foreign asset is represented by $n$ possible returns on the asset $\left\{\tilde{y}_{1}, \ldots, \tilde{y}_{n}\right\}$ and the associated second-order probability $\left\{q_{1}, \ldots, q_{n}\right\}$. In this

\footnotetext{
${ }^{11}$ For example, see Lewis (1999).

${ }^{12}$ Epstein and Miao (2003) explain the home bias puzzle under ambiguity within the framework of MEU.
} 
setting, the optimal portfolio allocation is determined by

$$
k^{*}=\operatorname{argmax}_{k} \sum_{\theta=1}^{n} q_{\theta} \phi\left(E\left[u\left((w-k) \tilde{x}+k \tilde{y}_{\theta}\right)\right]\right) .
$$

We assume that institutional investors usually estimate returns on assets from historical data and assign unique probability distributions to these returns. The optimal portfolio allocation is given by

$$
\left.k^{O}=\operatorname{argmax}_{k} E\left[u\left((w-k) \tilde{x}+k \tilde{y}^{O}\right)\right]\right) .
$$

Applying the result in the previous section to this setting, we find that $k^{O} \geq k^{*}$. That is, the individual investor purchases more of the domestic asset than the foreign asset compared with the optimal portfolio allocation derived by institutional investors. This is because an individual investor with insufficient information about the foreign asset takes into account the existence of ambiguity on the return on the foreign asset and she is likely to avoid to invest in the foreign asset. Note that Theorem 1 follows from the conditions under which the possible returns on an ambiguous asset are ranked by FSD and the Arrow-Pratt measure of relative risk aversion is less than 1. As pointed out by Meyer and Meyer (2005), it is unclear whether this condition is reasonable from an empirical viewpoint. As we mention in subsection 4.2 , however, by introducing the notion of MLRD, we derive the same result as Theorem 1 without assuming any condition on DM's utility function.

\section{Further Results}

In this section, we consider three further results as applications of the main result. First, for the purpose of extending the $50 \%$ rule for portfolio allocation problems, we consider conditions under which the $50 \%$ rule holds for the portfolio allocation problem with a risky asset and an ambiguous asset in the smooth ambiguity model. The $50 \%$ rule for portfolio allocation problems was investigated by Hadar and Seo (1988, 1990) and Clark and Jokung (1999). Second, by applying MLRD and RHRD to the analysis in the previous section, we analyze the effect of ambiguity on optimal portfolio allocation. Finally, we 
analyze the effect of ambiguity on the optimal portfolio allocation based on the notion of higher-order increases in risk by Ekern (1980).

\section{$4.1 \quad 50 \%$ Rule}

In this subsection, we investigate conditions under which the $50 \%$ rule holds for the portfolio allocation problem with a risky asset and an ambiguous asset. The so-called demand problem named by Kijima and Ohnshi (1996) has been paid attention to in the literature. Setting $w=1$, the portfolio allocation problem is formulated as follows:

$$
V(k)=\sum_{\theta=1}^{n} q_{\theta} \phi\left(E\left[u\left((1-k) \tilde{x}+k \tilde{y}_{\theta}\right)\right]\right) .
$$

Because $V^{\prime}(0)>0$ and $V^{\prime}(1)<0$, the optimal portfolio allocation $k^{*}$ is an interior solution in $[0,1]$.

Suppose that a risk averse investor can allocate her initial wealth to two risky assets that are independent and are equal in law, and suppose that her preferences are represented by the expected utility. Then, Rothschild and Stiglitz (1971) show that the optimal portfolio allocation is exactly equal to 0.5, as originally shown by Samuelson (1967). ${ }^{13}$ Conditions for the optimal portfolio allocation to be $k \leq 0.5$ have been investigated in the literature. This is called the $50 \%$ rule for portfolio allocation problems. ${ }^{14}$ By restricting the class of utility functions, Hadar and Seo (1988, Theorems 4 and 5) derive necessary and sufficient conditions for the optimal portfolio allocation to be $k \leq 0.5$. Clark and Jokung (1999) generalize Hadar and Seo (1988, Theorem 3) and derive sufficient conditions on the conditional distributions of the two risky assets under which the optimal portfolio allocation of one risky asset is less than 0.5 . For the purpose of extending the $50 \%$ rule for portfolio allocation

\footnotetext{
${ }^{13}$ Gollier (2001, Proposition 5) generalizes Rothschild and Stiglitz (1971) and shows that the optimal portfolio allocation for $n$ assets is perfectly diversified. That is, the portfolio consists of an equal amount of each asset.

${ }^{14}$ The previous studies examine conditions under which the optimal portfolio allocation for one asset is greater than $50 \%, k \geq 0.5$. Because it is essentially identical, their results are restated as $k \leq 0.5$, to agree with the settings in this paper.
} 
problems, based on the smooth ambiguity model, we investigate conditions under which the $50 \%$ rule holds for the portfolio allocation problem with a risky asset and an ambiguous asset. From the main result of this paper, we have

$$
V^{\prime}(0.5) \leq 0 \Leftrightarrow k^{*} \leq 0.5
$$

by putting $w=1$ and $k^{O}=0.5$ when the possible returns on the asset $\left\{\tilde{y}_{1}, \ldots, \tilde{y}_{n}\right\}$ are ranked by FSD and $R(z) \leq 1$. Recall that $R(z)=-z u^{\prime \prime}(z) / u^{\prime}(z)$ denotes the Arrow-Pratt measure of relative risk aversion. These are the conditions for the $50 \%$ rule for the portfolio allocation problem with a risky asset and an ambiguous asset. We summarize this argument in the following corollary.

Corollary 1. Suppose that an investor's objective function is represented by Equation (7), and that $\tilde{x} \stackrel{\mathrm{d}}{=} \sum_{\theta=1}^{n} q_{\theta} \tilde{y}_{\theta}$. The $50 \%$ rule holds, that is, $k^{*} \leq 0.5$ if the possible returns on the asset $\left\{\tilde{y}_{1}, \ldots, \tilde{y}_{n}\right\}$ are ranked by FSD and $R(z) \leq 1$.

This result can be applied to every compound return on the asset $\tilde{y}$ for which $k^{O}=0.5$. It can also be applied to other stochastic dominance relations mentioned in the following subsections by imposing appropriate conditions on the utility function $u$.

\subsection{Monotone Likelihood Ratio Dominance and Reversed Hazard Ratio Dominance}

When the possible returns on an ambiguous asset are ranked by FSD, we can conclude that ambiguity decreases the optimal portfolio allocation for investors whose Arrow-Pratt measure of relative risk aversion is less than unity. The condition, $R(z) \leq 1$, is assumed in determining the effect of FSD shifts in various decision problems. See, for example, Fishburn and Porter (1976) and Cheng et al. (1987). However, as pointed out by Meyer and Meyer (2005), it is unclear whether this condition is reasonable from an empirical viewpoint. We also question whether empirical observations under expected utility theory 
can be directly applied to the smooth ambiguity model even though $R(z) \leq 1$ is viewed as a reasonable property.

In this subsection, based on the motivations above, we introduce MLRD as a stronger notion of stochastic dominance than FSD. Let $\tilde{y}_{i}$ and $\tilde{y}_{j}$ be random variables for $i, j \in \Theta=\{1, \ldots, n\}$. Then, we say that $\tilde{y}_{j}$ is greater than $\tilde{y}_{i}$ in the sense of MLRD, denoted by $\tilde{y}_{i} \lesssim$ MLRD $\tilde{y}_{j}$, if $g_{j}(t) / g_{i}(t) \geq g_{j}(s) / g_{i}(s)$ for any $s, t \in[a, b]$ with $s<t$, where $G_{\theta}$ and $g_{\theta}$ denote the probability distribution function of $\tilde{y}_{\theta}$ and the probability density function of $\tilde{y}_{\theta}$ for $\theta \in \Theta$, respectively. Applying MLRD to rank the possible returns on assets, we obtain the same results as in the previous subsection with no restrictions on $u$. That is, we do not have to assume any conditions on $u$ such that $R(z)=-z u^{\prime \prime}(z) / u^{\prime}(z) \leq$ 1. To achieve this, we need results corresponding to Lemmas 2 and 3 for MLRD. ${ }^{15}$ Landsberger and Meilijson (1990) show that $k^{i} \leq k^{j}$ for $\tilde{y}_{i} \lesssim$ MLRD $\tilde{y}_{j}$. Recall that $k^{i}$ is defined by (1) for $i=1, \ldots, n$. Thus, we obtain the result corresponding to Lemma 3 by applying a similar argument. Kijima and Ohnishi (1996, Proposition 3.4) show that MLRD satisfies the convolution property for $\tilde{x}$, that is, $\tilde{x}+\tilde{y}_{i} \lesssim_{\text {MLRD }} \tilde{x}+\tilde{y}_{j}$ for any random variable $\tilde{x}$ such that $\tilde{x}$ and $\tilde{y}_{i}$ are independent and $\tilde{x}$ and $\tilde{y}_{j}$ are independent if the probability density function of $\tilde{x}$ is the Pòlya frequency function of order $2\left(\mathrm{PF}_{2}\right) .{ }^{16}$ As pointed out in Kijima and Ohnishi (1996, p.275), every $\mathrm{PF}_{2}$ function is characterized by the form $f(x)=e^{-\phi(x)}$, where $\phi(x)$ is a convex function. Furthermore, it can be shown that every $\mathrm{PF}_{2}$ function on $\mathbb{R}$ is log-concave. As in Lehmann (2005, p.323), the probability density functions of the normal distribution, the double exponential distribution, and the logistic distribution are $\mathrm{PF}_{2} \cdot{ }^{17}$ Therefore, the condition that the probability density function is $\mathrm{PF}_{2}$ is not restrictive. We obtain the following result by restricting our analysis to a class of probability density functions that are $\mathrm{PF}_{2}$.

Corollary 2. The existence of ambiguity aversion decreases the optimal portfolio allocation, $k^{*} \leq k^{O}$ if the possible returns on the asset $\left\{\tilde{y}_{1}, \ldots, \tilde{y}_{n}\right\}$ are

\footnotetext{
${ }^{15}$ For Lemmas 2 and 3, see Appendix B.

${ }^{16}$ For the definition of the Pòlya frequency function of order 2, see the Appendix.

${ }^{17}$ Note that their probability density functions are $f(x)=(1 / \sqrt{2 \pi \sigma}) e^{-(x-\mu)^{2} / 2 \sigma^{2}}, f(x)=$ $(1 / 2) e^{-|x|}$, and $f(x)=e^{-x} /\left(1+e^{-x}\right)^{2}$, respectively.
} 
ranked by $M L R D$ and their probability density functions are $P F_{2}$.

Next, we consider reversed hazard ratio dominance (RHRD) that is weaker than MLRD, which is shown, for example, in Eeckhoudt and Gollier (1995). ${ }^{18}$ Let $\tilde{y}_{i}$ and $\tilde{y}_{j}$ be random variables for $i, j \in \Theta=\{1, \ldots, n\}$. Then, we say that $\tilde{y}_{j}$ is greater than $\tilde{y}_{i}$ in the sense of RHRD, denoted by $\tilde{y}_{i} \lesssim$ RHRD $\tilde{y}_{j}$, if $G_{j}(t) / G_{i}(t) \geq g_{j}(t) / g_{i}(t)$ for any $t \in[a, b]$, where $G_{\theta}$ and $g_{\theta}$ denote the probability distribution function of $\tilde{y}_{\theta}$ and the probability density function of $\tilde{y}_{\theta}$ for $\theta \in \Theta$, respectively. ${ }^{19}$ Kijima and Ohnishi (1996, Theorem 3.3) show that $k^{i} \leq k^{j}$ for $\tilde{y}_{i} \lesssim_{\text {RHRD }} \tilde{y}_{j}$. Thus, we obtain the result corresponding to Lemma 3 by applying a similar argument. Kijima and Ohnishi (1996, Proposition 3.4) also show that RHRD satisfies the convolution property for $\tilde{x}$, that is, $\tilde{x}+$ $\tilde{y}_{i} \lesssim_{\mathrm{RHRD}} \tilde{x}+\tilde{y}_{j}$ for any random variable $\tilde{x}$ such that $\tilde{x}$ and $\tilde{y}_{i}$ are independent and $\tilde{x}$ and $\tilde{y}_{j}$ are independent if the probability density function of $\tilde{x}$ is the Pòlya frequency function of order $2\left(\mathrm{PF}_{2}\right)$. Thus, the following corollary is in order.

Corollary 3. The existence of ambiguity aversion decreases the optimal portfolio allocation, $k^{*} \leq k^{O}$ if the possible returns on the asset $\left\{\tilde{y}_{1}, \ldots, \tilde{y}_{n}\right\}$ are ranked by RHRD and their probability density functions are $P F_{2}$.

\subsection{Higher-Order Increases in Risk}

The notion of higher-order increases in risk is introduced by Ekern (1980), and has been analyzed, for example, by Eeckhdout and Shclesinger (2006) and Jindapon and Neilson (2007). In this subsection, we show that the result in this paper also applies to higher-order increases in risk.

For $\theta \in \Theta=\{1, \ldots, n\}$, let $G_{\theta}$ be probability distribution functions of random variables $\tilde{y}_{\theta}$ with supports contained in $[a, b]$. We define the functions

\footnotetext{
${ }^{18}$ In Eeckhoudt and Gollier (1995), RHRD is referred to as monotone probability ratio order.

${ }^{19}$ See Eeckhoudt and Gollier (1995, Lemma 2). Eeckhoudt and Gollier (1995, Lemma 1) also show that RHRD is stronger than FSD.
} 
by

$$
\begin{aligned}
& G_{\theta}^{1}(x)=G_{\theta}(x) \text { and } \\
& G_{\theta}^{k}(x)=\int_{a}^{x} G_{\theta}^{k-1}(t) d t
\end{aligned}
$$

for $x \in[a, b], \theta \in \Theta$, and $k=2, \ldots, N$, where the function $G_{\theta}^{n}$ denotes the $n$-th moment of $G_{\theta}$. The definition of the $N$-th degree increase in risk for $N \geq 2$ is as follows. Let $\tilde{y}_{i}$ and $\tilde{y}_{j}$ be random variables for $i, j \in \Theta$. Then, we say that $\tilde{y}_{j}$ is greater than $\tilde{y}_{i}$ in the sense of $N$-th degree risk, denoted by $\tilde{y}_{i} \lesssim_{\mathrm{N}-\text { risk }} \tilde{y}_{j}$, if $G_{j}^{N}(y) \geq G_{i}^{N}(y)$ and $G_{i}^{n}(b)=G_{j}^{n}(b)$ for $n=1, \ldots, N-1$. Note that if $\tilde{y}_{j}$ is greater than $\tilde{y}_{i}$ in the sense of $N$-th degree risk, then the first $(N-1)$-moments of $G_{i}$ and $G_{j}$ are equal. It is worth mentioning that $N=2$ corresponds to an increase in risk in the sense of Rothschild and Stiglitz (1970), and $N=3$ corresponds to an increase in downside risk in the sense of Menezes et al. (1980).

We need results corresponding to Lemmas 2 and 3 to obtain comparative statics results. The result corresponding to Lemma 3 is straightforward because it is shown by Chiu et al. (2010) that $k^{i} \leq k^{j}$ if $\tilde{y}_{i} \lesssim$ N-risk $\tilde{y}_{j}$, $(-1)^{n} u^{n}(x) \leq 0$ for $n=N, N+1$, and $-x u^{N+1}(x) / u^{N}(x) \leq N$, where the utility function $u$ is assumed to be strictly increasing and infinitely continuously differentiable, and $u^{n}$ denotes the $n$-th derivative of $u$. Recall that $k^{i}$ is defined by (1). The convolution property ${ }^{20}$ must hold to obtain the result corresponding to Lemma 2. In the following, we show that the convolution property holds. Because $\tilde{x}$ and $\tilde{y}_{\theta}$ are independent for any $\theta \in \Theta$, the convolution of $F$ and $G_{\theta}$ is ${ }^{21}$

$$
H_{\theta}(z)=\int_{a}^{b} G_{\theta}(z-x) d F(x),
$$

where $F$ and $G_{\theta}$ denote the probability distribution functions of $\tilde{x}$ and $\tilde{y}_{\theta}$, respectively. It can be shown that the convolution $H$ is also a probability

\footnotetext{
${ }^{20}$ Recall that the definition of the convolution property is in the Appendix.

${ }^{21}$ For the definition of the convolution of two probability distribution functions, see the Appendix. Note that the convolution property and the convolution of two probability distribution functions are different. See the Appendix.
} 
distribution function. Let us define $G_{\theta}^{n}(y-x)=\int_{a}^{y} G_{\theta}^{n-1}(t-x) d t$. By Fubini's theorem, we can rewrite the probability distribution function as

$$
H_{\theta}^{n}(z)=\int_{a}^{b} G_{\theta}^{n}(z-x) d F(x)
$$

Note that $\tilde{y}_{i} \lesssim_{n-\text { risk }} \tilde{y}_{j}$ is equivalent to $H_{i}^{n}(z)=\int_{a}^{b} G_{i}^{n}(z-x) d F(x) \geq \int_{a}^{b} G_{j}^{n}(z-$ $x) d F(x)=H_{j}^{n}(z)$, that is, $\tilde{x}+\tilde{y}_{i} \lesssim_{\text {n-risk }} \tilde{x}+\tilde{y}_{j}$. From the convolution property, we obtain the result corresponding to Lemma 2 . Hence, the following corollary is in order.

Corollary 4. The existence of ambiguity aversion decreases the optimal portfolio allocation, $k^{*} \leq k^{O}$ if the possible returns on the asset $\left\{\tilde{y}_{1}, \ldots, \tilde{y}_{n}\right\}$ are ranked by $N$-th degree risk, $(-1)^{n} u^{n}(x) \leq 0$ for $n=N, N+1$, and $-x u^{N+1}(x) / u^{N}(x) \leq N$.

\section{Conclusion}

This paper considers a portfolio allocation problem between a risky asset and an ambiguous asset. We determine conditions under which an investor decreases the optimal portfolio allocation for the ambiguous asset. The conditions are imposed on the investor's utility function $u$ and the stochastic dominance relations of $\left\{\tilde{y}_{1}, \ldots, \tilde{y}_{n}\right\}$. For FSD, the investor with an ArrowPratt measure of relative risk aversion less than unity decreases the portfolio allocation of the risky asset when ambiguity is incorporated into the model. Our analyses can be applied to an international diversification problem where our result provides a potential explanation of the home bias puzzle. Furthermore, as corollaries of the main result in this paper, we extend the $50 \%$ rule for portfolio allocation problems by Hadar and Seo $(1988,1990)$ and Clark and Jokung (1999) based on the smooth ambiguity model. Finally, we investigate the effect of ambiguity on the optimal portfolio allocation based on MLRD, RHRD, and higher-order increases in risk introduced Ekern by (1980).

This paper assumes that the return on the risky asset $\tilde{x}$ and the possible returns on the ambiguous asset $\tilde{y}_{\theta}$ are independent. This assumption enables us 
to apply the convolution property to our analyses. However, it is appropriate to assume that these assets are dependent. We leave this extension for future research. 


\section{Appendix}

\section{Appendix A}

We provide a definition of convolution in probability theory based on Billingsley (1995, p.266), Lehmann (2005, p.103), and Kijima and Ohnishi (1996, Appendix B), and provide some definitions from the theory of total positivity.

Definition 1. Let $\tilde{x}$ and $\tilde{y}$ be independent random variables with probabilities $\mu$ and $v$, respectively, and let $P$ and $Q$ be the corresponding probability distribution functions. The convolution of $P$ and $Q$ is defined by

$$
H(z) \equiv \int_{a}^{b} Q(z-x) d P(x) .
$$

It can be shown that $H$ is a probability distribution function. It can also be shown that if two random variables $\tilde{x}$ and $\tilde{y}$ with probability distribution functions $P$ and $Q$ are independent, then $\tilde{x}+\tilde{y}$ has the probability distribution function $H$ defined by (8).

Next, we introduce the convolution property.

Definition 2. A stochastic order $\lesssim$ st satisfies the convolution property if $\tilde{x}+$ $\tilde{y}_{i} \lesssim_{\text {st }} \tilde{x}+\tilde{y}_{j}$ for any random variable $\tilde{x}$ such that $\tilde{x}$ and $\tilde{y}_{i}$ are independent and $\tilde{x}$ and $\tilde{y}_{j}$ are independent.

As in Kijima and Ohnishi (1996, Proposition 3.3), FSD satisfies the convolution property. However, MLRD does not necessarily satisfy the convolution property. Proposition 3.4 in Kijima and Ohnishi (1996) shows that MLRD and RHRD satisfy the convolution property for $\tilde{x}$ if the probability density function of $\tilde{x}$ is Pòlya frequency function of order $2\left(\mathrm{PF}_{2}\right)$. The definition of $\mathrm{PF}_{2}$ functions is based on the notion of being totally positive of order $2\left(\mathrm{TP}_{2}\right)$, so we first define $\mathrm{TP}_{2}$ functions.

Definition 3. A function $K: \mathbb{R} \times \mathbb{R} \rightarrow \mathbb{R}_{+}$is totally positive of order $2\left(\mathrm{TP}_{2}\right)$, denoted by $K \in \mathrm{TP}_{2}$ if for $x_{1}<x_{2}$ and $y_{1}<y_{2}$,

$$
K\left(x_{1}, y_{1}\right) K\left(x_{2}, y_{2}\right)-K\left(x_{1}, y_{2}\right) K\left(x_{2}, y_{1}\right) \geq 0
$$


As pointed out in Jewitt (1987, p.77), the function $K(x, y)=e^{x y}$ is $\mathrm{TP}_{2}$, and any probability density function of the form $f(x, y)=\gamma(x) \psi(y) e^{x y}$ is $\mathrm{TP}_{2}$. Therefore, the probability density functions of the nomal distribution, the exponential distribution, the binomial distribution, and the Poisson distribution are $\mathrm{TP}_{2}$. Finally, we provide the definition of $\mathrm{PF}_{2}$ functions.

Definition 4. A non-negative function $f(x)$ is Pòlya frequency function of order $2\left(\mathrm{PF}_{2}\right)$ in $x$ if $f(x-y)$ is $\mathrm{TP}_{2}$ in $x$ and $y$; i.e.,

$$
f\left(x_{1}-y_{1}\right) f\left(x_{2}-y_{2}\right) \geq f\left(x_{1}-y_{2}\right) f\left(x_{2}-y_{1}\right), x_{1}<x_{2}, y_{1}<y_{2} .
$$

\section{Appendix B. Proof of Theorem 1}

We now prove Theorem 1 using the technique of the monotone comparative statics under uncertainty developed by Jewitt (1987) and Athey (2002).

Definition 5. A real-valued function $g: \mathbb{R} \rightarrow \mathbb{R}$ satisfies the single crossing condition from below if there exists some $\bar{x}$ such that $(x-\bar{x}) g(x) \geq 0$ for any $x$.

The following notion is equivalent to being $\mathrm{TP}_{2}$, which is defined in Definition 3.

Definition 6. A function $K: \mathbb{R} \times \mathbb{R} \rightarrow \mathbb{R}_{+}$is log-supermodular if for $x_{1}<x_{2}$ and $y_{1}<y_{2}$,

$$
K\left(x_{1}, y_{1}\right) K\left(x_{2}, y_{2}\right)-K\left(x_{1}, y_{2}\right) K\left(x_{2}, y_{1}\right) \geq 0
$$

Because "log-supermodularity" is more widely used than " $\mathrm{TP}_{2}$ " in economics, we use "log-supermodularity" in this paper. Log-supermodularity is characterized by the following lemma. See, for example, Topkis (1978), Kijima and Ohnishi (1996, p.274) or Gollier (2001).

Lemma 1. Suppose that $K: \mathbb{R} \times \mathbb{R} \rightarrow \mathbb{R}_{+}$is twice continuously differentiable. Then, $K$ is log-supermodular if and only if one of the following two equivalent conditions holds:

(1) For any $x, x_{0} \in \mathbb{R}$ and for any $y_{1}<y_{2}$,

$$
\left(x-x_{0}\right) \frac{K\left(x, y_{2}\right)}{K\left(x_{0}, y_{2}\right)} \geq\left(x-x_{0}\right) \frac{K\left(x, y_{1}\right)}{K\left(x_{0}, y_{1}\right)}
$$


(2) $(\partial K(x, y) / \partial x) / K(x, y)$ is non-decreasing in $y$. Equivalently, $\partial^{2} K(x, y) / \partial x \partial y \geq$ 0 .

Lemma 2. For $i, j \in \Theta=\{1, \ldots, n\}$ with $i<j$,

$$
E\left[u\left((w-k) \tilde{x}+k \tilde{y}_{i}\right)\right] \leq E\left[u\left((w-k) \tilde{x}+k \tilde{y}_{j}\right)\right] .
$$

Proof. Because FSD satisfies the convolution property, it follows that $\tilde{x}+$ $\tilde{y}_{i} \lesssim_{\mathrm{FSD}} \tilde{x}+\tilde{y}_{j}$ for $i<j$ and for any random variable $\tilde{x}$ such that $\tilde{x}$ and $\tilde{y}_{i}$ are independent and $\tilde{x}$ and $\tilde{y}_{j}$ are independent. By FSD, it follows that $E\left[u\left((w-k) \tilde{x}+k \tilde{y}_{i}\right)\right] \leq E\left[u\left((w-k) \tilde{x}+k \tilde{y}_{j}\right)\right]$, which proves the claim.

Furthermore, log-supermodularity is also characterized by the following theorem. See, for example, Karlin (1968), Jewitt (1987), Athey (2002), or Gollier (2001).

Theorem 2. Let $K: \mathbb{R} \times \mathbb{R} \rightarrow \mathbb{R}_{+}$be a non-negative real-valued function. Suppose that the function $g: \mathbb{R} \rightarrow \mathbb{R}$ satisfies the single crossing condition from below. Then, for any random variable $\tilde{x}$ and any $y_{1}<y_{2}$,

$$
E\left[g(\tilde{x}) K\left(\tilde{x}, y_{1}\right)\right]=0 \Rightarrow E\left[g(\tilde{x}) K\left(\tilde{x}, y_{2}\right)\right] \geq 0
$$

if and only if $K$ is log-supermodular.

Theorem 3. (Hadar and Seo (1990)) Suppose that a) $\left.u^{\prime}>0, u^{\prime \prime} \leq 0, b\right) \tilde{x}^{i}$ and $\tilde{y}$ are independent for $i=1,2$, and c) $E\left[u\left(\left(w-k_{i}\right) \tilde{x}^{i}+k_{i} \tilde{y}\right)\right]$ is maximized at $k_{i}^{*}$. Then, $k_{1}^{*} \leq k_{2}^{*}$ for any $\tilde{x}^{2} \lesssim_{\mathrm{FSD}} \tilde{x}^{1}$ if and only if $u^{\prime}(z) z$ is non-decreasing if and only if $R(z) \leq 1$.

Based on Theorem 3, we can prove the following lemma, which plays a key role in proving Theorem 1.

Lemma 3. Let $g(\theta)=E\left[\left(\tilde{y}_{\theta}-\tilde{x}\right) u^{\prime}\left(\left(w-k^{*}\right) \tilde{x}+k^{*} \tilde{y}_{\theta}\right)\right]$ for any $\theta \in \Theta=$ $\{1, \ldots, n\}$. Then, $g$ satisfies the single crossing condition from below.

Proof. It follows from Theorem 3 that $k^{j} \leq k^{i}$ for $\tilde{y}_{i} \lesssim_{\text {FSD }} \tilde{y}_{j}$ for $i, j \in \Theta=$ $\{1, \ldots, n\}$ with $i<j$ if and only if $R(z)=-z u^{\prime \prime}(z) / u(z) \leq 1$. This implies 
that $k^{1} \leq \ldots \leq k^{n}$. Because $k^{1} \leq k^{*} \leq k^{n}$, there exists some $i \in \Theta$ such that $k^{i-1} \leq k^{*} \leq k^{i}$. By the concavity, we have

$$
\begin{aligned}
& E\left[\left(\tilde{y}_{\theta}-\tilde{x}\right) u^{\prime}\left(\left(w-k^{*}\right) \tilde{x}+k^{*} \tilde{y}_{\theta}\right)\right] \leq 0 \text { for } \theta \leq i \text { and } \\
& E\left[\left(\tilde{y}_{\theta}-\tilde{x}\right) u^{\prime}\left(\left(w-k^{*}\right) \tilde{x}+k^{*} \tilde{y}_{\theta}\right)\right] \geq 0 \text { for } \theta>i,
\end{aligned}
$$

which proves the lemma.

Proof of Theorem 1. Let $v(\theta)=E\left[u\left(\left(w-k^{*}\right) \tilde{x}+k^{*} \tilde{y}_{\theta}\right)\right]$, let $g(\theta)=E\left[\left(\tilde{y}_{\theta}-\right.\right.$ $\left.\tilde{x}) u^{\prime}\left(\left(w-k^{*}\right) \tilde{x}+k^{*} \tilde{y}_{\theta}\right)\right]$ for any $\theta \in \Theta=\{1, \ldots, n\}$, and let $\phi(v(\theta), i)=$ $\phi_{i}(v(\theta))$ for $i=1,2$. For ease of exposition, let $i=2$ correspond to ambiguity neutrality (that is, $\phi_{2}(v(\theta))$ is linear), and let $i=1$ correspond to ambiguity aversion (that is, $\phi_{1}(v(\theta))$ is concave). It follows from Lemmas 1 and 2 that $\phi^{\prime}(v(\theta), i)=\partial \phi(v(\theta), i) / \partial v(\theta)$ is log-supermodular if and only if $\phi_{1}$ is more ambiguity averse than $\phi_{2}$. Because $g(\theta)$ satisfies the single crossing condition from below by Lemma 3 , it follows that

$$
\begin{aligned}
& \begin{array}{c}
\phi_{1} \text { is more ambiguity averse than } \phi_{2} \\
\Leftrightarrow
\end{array} \\
\Leftrightarrow & \sum_{\theta=1}^{n} q_{\theta} \phi_{1}^{\prime}\left(E\left[u\left(\left(w-k^{*}\right) \tilde{x}+k^{*} \tilde{y}_{\theta}\right)\right]\right) g(\theta)=0 \\
\Rightarrow & \sum_{\theta=1}^{n} q_{\theta} \phi_{2}^{\prime}\left(E\left[u\left(\left(w-k^{*}\right) \tilde{x}+k^{*} \tilde{y}_{\theta}\right)\right]\right) g(\theta) \geq 0 \\
\Leftrightarrow & \sum_{\theta=1}^{n} q_{\theta} g(\theta) \geq 0 \\
\Leftrightarrow & \sum_{\theta=1}^{n} q_{\theta} E\left[\left(\tilde{y}_{\theta}-\tilde{x}\right) u^{\prime}\left(\left(w-k^{*}\right) \tilde{x}+k^{*} \tilde{y}_{\theta}\right)\right] \geq 0,
\end{aligned}
$$

where the first equivalence follows from Theorem 2, the second equivalence follows from the linearity of $\phi_{2}(\cdot)$ and $\phi_{2}^{\prime}(\cdot)>0$, and the last equivalence follows from the definition of $g(\theta)$. This completes the proof. 


\section{References}

[1] Arrow, K. J. (1965): Aspects of the Theory of Risk-Bearing, Helsinki: Yrjo Jahnsonin Saatio.

[2] Athey, S. (2002): "Monotone Comparative Statics under Uncertainty," Quarterly Journal of Economics 117, 187-223.

[3] Billingsley, P. (1995): Probability and Measure, 3rd edition, Wiley.

[4] Cheng, H. C., M. J. Magill and W. J. Shafer (1987): "Some Results on Comparative Statics under Uncertainty," International Economic Review 28, 493-507.

[5] Chiu, W. H., L. Eeckhoudt and B. Rey (2012): "On Relative and Partial Risk Attitudes: Theory and Implications," Economic Theory 50, 151-167.

[6] Clark, E., and O. Jokung (1999): "A Note on Asset Proportions, Stochastic Dominance, and the 50\% Rule," Management Science 45, 1724-1727.

[7] Ekern, S. (1980): "Increasing Nth Degree Risk," Economics Letters 6, 329-333.

[8] Eeckhoudt, L. and C. Gollier (1995): "Demand for Risky Assets and the Monotone Probability Ratio Order," Journal of Risk and Uncertainty 11, 113-122.

[9] Eeckhoudt, L. and H. Schlesinger, (2006): "Putting Risk in its 'Proper Place," American Economic Review 96, 280-289.

[10] Ellsberg, D. (1961): "Risk, Ambiguity, and the Savage Axioms," Quarterly Journal of Economics 75, 643-669.

[11] Epstein, L. G. and J. Miao (2003): "A Two-Person Dynamic Equilibrium under Ambiguity," Journal of Economic Dynamics and Control 27, 12531288. 
[12] Fishburn, P. C. and R. B. Porter (1976): "Optimal Portfolios with One Safe and One Risky Asset: Effects of Changes in Rate of Return and Risk," Management Science 22, 1064-1073.

[13] French, K. R. and J. M. Poterba (1991): "Investor Diversification and International Equity Markets," American Economic Review 81, 221-226.

[14] Gilboa, I. and D. Schmeidler (1989): "Maxmin Expected Utility with Non-Unique Priors," Journal of Mathematical Economics 18, 141-153.

[15] Gollier, C. (2001): The Economic of Risk and Time, MIT Press.

[16] Gollier, C. (2011): "Portfolio Choice and Asset Prices: the Comparative Statics of Ambiguity Aversion," Review of Economic Studies 78, 13291344.

[17] Hadar, J. and T. K. Seo (1988): "Asset Propotions in Optimal Portfolios," Review of Economic Studies 55, 459-468.

[18] Hadar, J. and T. K. Seo (1990): "The Effects of Shifts in a Return Distribution on Optimal Portfolios," International Economic Review 31, 721736.

[19] Huang, Y-C, and L. Y. Tzeng (2017): "A Mean-preserving Increase in Ambiguity and Portfolio Choices," forthcoming in Journal of Risk and Insurance.

[20] Jewitt, I. (1987): "Risk Aversion and the Choice between Risky Prospects: The Preservation of Comparative Static Results," Review of Economic Studies 53, 73-85.

[21] Jindapon, P. and W. S. Neilson (2007): "Higher-order Generalizations of Arrow-Pratt and Ross Risk Aversion: A Comparative Statics Approach," Journal of Economic Theory 136, 719-728.

[22] Karlin, S. (1968): Total Positivity, Stanford, Stanford University Press.

[23] Keynes, J. M. (1921): A Treatise on Probability, London: MacMillan. 
[24] Kijima, M. and M. Ohnishi (1996): "Portfolio Selection Problems via the Bivariate Characterization of Stochastic Dominance Relations," Mathematical Finance 6, 237-277.

[25] Klibanoff, P., M. Marinacci, and S. Mukerji (2005): "A Smooth Model of Decision Making under Ambiguity," Econometrica 73, 1849-1892.

[26] Knight, F. H. (1921). Risk, Uncertainty and Profit, Hart, Schaffner and Marx.

[27] Landsberger, M. and I. Meilijson (1990): "Demand for Risky Financial Assets: A Portfolio Analysis," Journal of Economic Theory 50, 204-213.

[28] Lehmann, E. L. (2005): Testing Statistical Hypotheses, 3rd edition, Springer.

[29] Levy, H. (1992): "Stochastic Dominance and Expected Utility: Survey and Analysis," Management Science 38, 555-593.

[30] Lewis, K. K. (1999): "Trying to Explain Home Bias in Equities and Consumption," Journal of Economic Literature 37, 571-608.

[31] Menezes, C., C. Geiss and J. Tressler (1980): "Increasing Downside Risk," American Economic Review 70, 921-932.

[32] Meyer, D. J. and J. Meyer (2005): "Relative Risk Aversion: What do We Know?," Journal of Risk and Uncertainty 31, 243-262.

[33] Osaki, Y. and H. Schlesinger (2014): "Portfolio Choice and Ambiguous Backgrou Risk," Working Paper, University of Alabama. Available at http://hschlesinger.people.ua.edu/current-working-papers.html

[34] Pratt, J. W. (1964): "Risk Aversion in the Small and the Large," Econometrica $32,122-136$.

[35] Rothschild, M. and J. E. Stiglitz (1970): "Increasing Risk: I. A Definition," Journal of Economic Theory 2, 225-243. 
[36] Rothschild, M. and J. E. Stiglitz (1971): "Increasing Risk: II. Its Economic Consequences," Journal of Economic Theory 3, 66-84.

[37] Samuelson, P. A. (1967): "General Proof That Diversification Pays," Journal of Financial and Quantitative Analysis 2, 1-13.

[38] Schmeidler, D. (1989): "Subjective Probability and Expected Utility without Additivity," Econometrica 57, 571-587.

[39] Segal, U. (1987): "The Ellsberg Paradox and Risk Aversion: An Anticipated Utility Approach," International Economic Review 28, 175-202.

[40] Strzalecki, T. (2013): "Temporal Resolution of Uncertainty and Recursive Models of Ambiguity Aversion," Econometrica 81, 1039-1074.

[41] Topkis, D. (1978): "Minimizing a Submodular Function on a Lattice," Operations Research 26, 305-321. 